\title{
IMPLICIT CONTROLLER FOR CLASSES OF NONLINEAR SYSTEMS
}

\author{
S. S. Ge ${ }^{*}$ and M.W. Spong** \\ * Department of Electrical and Computer Engineering, \\ National University of Singapore, Singapore 117576 \\ Email: elegesz@nus.edu.sg \\ ** Coordinated Science Lab, \\ University of Illinois at Urbana-Champaign, \\ Urbana, IL 61801, USA \\ Email: m-spong@uiuc.edu
}

\begin{abstract}
In this paper, implicit controls are investigated for different classes of nonlinear systems, nonaffine systems in pure-feedback form and affine systems in strict-feedback form. While control system design for general unknown nonaffine nonlinear systems needs further investigation, elegant control designs are presented for known systems and systems with unknown scalar parametrization. Though for affine nonlinear systems, it can be shown that the resulting control laws are equivalent to that obtainable based on feedback linearization control, the approaches are fundamentally different. The most important differences/features are that (i) the implicit control is a function of both states and control signals, rather than states alone, and (ii) the resulting sampled-data systems are different, subsequently, their stability results are different. Simulation studies are carried out to show the validity of the proposed design method. Copyright (C)2005 IFAC
\end{abstract}

Keywords: Implicit control, sampled-data systems, hybrid systems

\section{INTRODUCTION}

Adaptive control of nonlinear systems using feedback linearization has been extensively studied for affine nonlinear systems. However, for many practical systems, the input variables may not be in an affine form. Due to the lack of mathematical tools, control of nonaffine nonlinear systems is difficult and challenging. In fact, it is impossible to handle the control problem of the nonaffine nonlinear system directly because, in general, even if it is known that the inverse exists, it is impossible to construct it analytically. Consequently, no control system design is possible along the lines of conventional model-based control (Ge et al., 1999a),(Hovakimyan et al., 2002). In (Nesic et al., 1999), other possible difficulties in dealing with non-affine systems in general are addressed. The authors showed that in general there may not exist a continuous controller for non-affine systems that yields stable zero dynamics, but a discontinuous controller may be obtained.

For nonaffine nonlinear systems, several researchers have suggested to use neural networks as emulators of inverse systems (Jin et al., 1993),(Levin and Narendra, 1996). Based on implicit function theory, the NN control methods proposed in (Jin et al., 1993),(Goh, 1994),(Goh and Lee, 1994) have been used to emulate the "inverse controller" to achieve tracking control objectives. Nevertheless, no rigorous stability proofs of the closedloop systems were given for on-line adaptive NN control due to the difficulties in analysis. In (Ge et 
al., 1999a),(Ge et al., 1999b), stable adaptive NN control was constructed for nonaffine nonlinear systems using the Implicit Function Theorem and the Mean Value Theorem that are not usually associated with neural network control theory. In (Hovakimyan et al., 2002), under the assumption of the existence of an linearizing control, approximated linearizing feedback control is constructed for a class of nonaffine nonlinear systems with the lower bound of the estimate of the control effectiveness being explicitly given.

The pure-feedback system represents a more general class of triangular systems for which no affine appearance of the variables can be used as virtual controls. As indicated in (Krstić et al., 1995), it is quite restrictive to find the explicit virtual controls to stabilize the pure-feedback systems by using integrator backstepping. There are only a handful of results available in the literature using neural networks to emulate the inverse with either very strict restrictions or without rigorous stability analysis. Only a few results are available in the literature for the control of nonlinear pure-feedback systems. In (Kanellakipoulos et al., 1991), (Krstić et al., 1995), only local stability is achieved in a well defined region around the origin for parametric pure-feedback systems. By imposing additional restrictions on the nonlinearities, global stability is obtained for a special case of the parametric pure-feedback systems in (Seto et al., 1994). Note that in these papers, the nonlinearities are known smooth functions, and the unknown parameters occur linearly. In (Simoes et al., 1996), implicit control laws that are not necessarily smooth are proposed for nonlinear purefeedback systems in discrete-time with dynamics that are completely known.

In this paper, we consider the control system design based on the explicit construction of implicit control in continuous-time, and which is readily applicable to hybrid system implementation (e.g. sampled data systems). Furthermore, we integrate our implicit control approach with adaptive control technique to add robustness to parameter uncertainties. The advantages of our approach are that (i) stable control system design can be easily constructed by exploiting the idea of implicit control system design for general nonaffine nonlinear systems with known dynamics, (ii) more flexibility is introduced by allowing the regressor to depends on both $x$ and $u$, which makes it more straightforward to exploit the linearity in the parameters assumption, (iii) the control system is free from control singularity problem that appears in adaptive control for affine nonlinear systems using feedback linearization control, and (iv) the increase in the basin of attraction.

\section{PROBLEM FORMULATION}

In this paper, consider the following class of nonaffine nonlinear systems, i.e., nonlinear purefeedback systems as

$$
\begin{aligned}
& \dot{x}_{i}=f_{i}\left(\bar{x}_{i}, x_{i+1}\right), \quad 1<i \leq n-1 \\
& \dot{x}_{n}=f_{n}(x, u)
\end{aligned}
$$

where $\bar{x}_{i}=\left[x_{1}, \cdots, x_{i}\right]^{T} \in R^{i}, x=\left[x_{1}, \cdots, x_{n}\right]^{T} \in$ $R^{n}, u \in R$ are the state variables, and system input respectively. $f_{i}(\cdot, \cdot), i=1, \ldots, n$ are unknown smooth functions.

Assumption 1. $f_{i}\left(\bar{x}_{i}, x_{i+1}\right), i=1, \ldots, n-1$, are $C^{1}$ for $\left(\bar{x}_{i}, x_{i+1}\right) \in R^{i+1}$ and $f_{n}(x, u)$ are $C^{1}$ for $(x, u) \in R^{n+1}$.

Assumption 2. $\partial f_{i}\left(\bar{x}_{i}, x_{i+1}\right) / \partial x_{i+1} \neq 0, \forall\left(\bar{x}_{i}, x_{i+1}\right)$ $\in R^{i+1}$ and $\partial f_{n}(x, u) / \partial u \neq 0, \forall(x, u) \in R^{n+1}$.

Remark 1. System (1) covers a large class of nonlinear systems, and includes the class of affine systems, systems in strict-feedback forms as special cases. Though intensive research has been carried out for systems of an affine form, their results cannot be applied to nonaffine systems. Even if the description of $f(x, u)$ is known exactly, it is not easy to design a explicit feedback control for achieving feedback linearization. When the structure of $f(x, u)$ is unknown it is even more difficult to construct the controller.

\section{NONAFFINE NONLINEAR SYSTEMS IN THE LINEAR-IN-THE-PARAMETERS FORM}

In this section, let us investigate the class of nonaffine nonlinear systems (1) whose nonlinear functions are in the linear-in-the-parameters (LIP) form, i.e., $f_{i}\left(\bar{x}_{i}, x_{i+1}\right)=\theta_{i}^{T} \psi_{i}\left(\bar{x}_{i}, x_{i+1}\right), 1 \leq i \leq$ $n-1$, and $f_{n}(x, u)=\theta_{n}^{T} \psi_{n}(x, u)$.

\subsection{First-Order Nonaffine Systems - Ideal Case}

It can easily be seen that control system design can be conveniently carried out, which may otherwise be difficult, as we are not seeking for the construction of an explicit inverse controller. Inverse control is carried out implicitly as will be demonstrated. To show the idea clearly, let us consider the first order nonaffine nonlinear systems

$$
\dot{x}_{1}=f_{1}\left(x_{1}, u_{1}\right)
$$

where $x_{1} \in R$ and $u_{1} \in R$ are the state and input respectively, and $f_{1}\left(x_{1}, u_{1}\right)=\theta_{1}^{T} \psi_{1}\left(x_{1}, u_{1}\right) \in R$ is a nonaffine function of both $x_{1}$ and $u_{1}$, with 
$\theta_{1} \in R^{l_{1}}$ and $\psi_{1}\left(x_{1}, u_{1}\right) \in R^{l_{1}}$ being dimensionally compatible constant parameters and known regressor, respectively.

Remark 2. It is interesting to note that more flexibility is introduced by allowing the regressor to depend on both $x_{1}$ and $u_{1}$, and this makes it more straightforward to exploit the linearity in the parameters assumption. For example, $f_{1}\left(x_{1}, u_{1}\right)=$ $a_{1} x_{1}^{2}+a_{2} u_{1}^{3}+a_{3} \sin \left(u_{1}\right)$ can be conveniently expressed as $f_{1}\left(x_{1}, u_{1}\right)=\theta_{1}^{T} \psi_{1}\left(x_{1}, u_{1}\right)$ where $\theta_{1}=$ $\left[a_{1}, a_{2}, a_{3}\right]^{T}$ and $\psi_{1}\left(x_{1}, u_{1}\right)=\left[x_{1}^{2}, u_{1}^{3}, \sin \left(u_{1}\right)\right]^{T}$.

Note that even for the ideal case when $\theta_{1}$ and $\psi_{1}$ are known, it is difficult, if not impossible, to obtain an explicit expression of the control for a general nonaffine nonlinear function. As such, the control problem is by no means trivial.

To solve the problem, add and subtract $b u_{1}$ on the right hand side of equation (2), we have

$$
\dot{x}_{1}=\left[\theta_{1}^{T} \psi_{1}\left(x_{1}, u_{1}\right)-b_{1} u_{1}\right]+b_{1} u_{1}
$$

Consider the implicit control $u_{1}$ given by

$$
u_{1}=\frac{1}{b_{1}}\left(-\theta_{1}^{T} \psi_{1}\left(x_{1}, u_{1}\right)-k_{1} x_{1}\right)+u_{1}
$$

where $k_{1}>0$ and $b_{1}$ is a design constant. From (4), we know that $u_{1}$ is actually the solution of

$$
\theta_{1}^{T} \psi_{1}\left(x_{1}, u_{1}\right)+k_{1} x_{1}=0
$$

In theory, the existence of the solution for $u_{1}$ is guaranteed provided that $\frac{\partial\left[\theta_{1}^{T} \psi_{1}\left(x_{1}, u_{1}\right)\right]}{\partial u_{1}} \neq 0$. As $\frac{\partial f_{1}\left(x_{1}, u_{1}\right)}{\partial u_{1}}=\frac{\partial\left[\theta_{1}^{T} \psi_{1}\left(x_{1}, u_{1}\right)\right]}{\partial u_{1}} \neq 0$, which is actually the controllability condition, we know that the solution for $u_{1}$ of equation (5) always exists.

Accordingly, we have $\dot{x}_{1}=-k x_{1}$, which shows that the closed-loop system is stable and $x_{1}(t)=$ $e^{-k t}+x_{1}(0)$ will exponentially converge to zero when $t$ tends to infinity.

Theorem 1. For system (2) satisfying Assumptions 1 and 2, implicit control (4) guarantees that $x_{1} \rightarrow 0$ as $t \rightarrow \infty$ and the control signal $u_{1}$ is bounded in the closed-loop.

In addition, the advantage of the proposed implicit control can be seen clearly - even for the ideal case, the explicit solution of equation (5) is difficult to obtain, while it could be easily solved by (4) in digital implementation. In the sequel, we will discuss the issue of stability pertaining to digital implementation.

Remark 3. Note that $b_{1}$ is an artificial constant introduced by the designer for generality. For the implicit control (4), it seems to be of no importance. However, it plays an important role for the corresponding digital implementation for sampled-data systems as will be demonstrated later.

\subsection{Higher-Order Nonaffine Systems - Ideal Case}

For the pure-feedback system (1), the control problem can be conveniently solved by combining backstepping and the newly introduced implicit control design.

The design method for the higher-order systems is essentially the same as for the first-order counterpart except that in the intermediate design steps, the systems' states will be artificially introduced to compensate for the lack of affine terms, which is usually used to construct virtual controls in the backstepping design.

For clarity, let us define the Lyapunov function candidate $V_{i}$, the intermediate control functions $\alpha_{i}$, and the control law as

$$
\begin{aligned}
& V_{i}=V_{i-1}+\frac{1}{2} z_{i}^{2}, \quad 1 \leq i \leq n \\
& \alpha_{i}=\frac{1}{b_{i}}\left[-b_{i-1} z_{i-1}-k_{i} z_{i}-\theta_{i, a}^{T} \psi_{i, a}\right]+x_{i+1}, \\
& \quad 1 \leq i \leq n-1 \\
& u=\frac{1}{b_{n}}\left[-b_{n-1} z_{n-1}-k_{n} z_{n}-\theta_{n, a}^{T} \psi_{n, a}\right]+u
\end{aligned}
$$

where $\theta_{i, a}=\left[\theta_{i}^{T}, \ldots, \theta_{1}^{T}\right]^{T}$, and

$$
\psi_{i, a}=\left[\left(1-\xi_{i}\right) \psi_{i}^{T},-\xi_{i-1} \psi_{i-1}^{T}, \ldots,-\xi_{1} \psi_{1}^{T}\right]^{T}
$$

with $\xi_{j}:=\frac{\partial \alpha_{i-1}}{\partial x_{j}}, V_{0}=0, \theta_{1, a}=\theta_{1}, \psi_{1, a}=\psi_{1}$, $\alpha_{0}=0$, and $z_{0}=0$.

Step $i(1<i \leq n-1)$. Rewriting the $z_{i}$ subsystem as $z_{i}=x_{i}-\alpha_{i-1}, \dot{z}_{i}=\dot{x}_{i}-\dot{\alpha}_{i-1}$, and $z_{i+1}=x_{i+1}-\alpha_{i}$, and noting that $\alpha_{i-1}$ is the function of $\bar{x}_{i}$, we have

$$
\begin{aligned}
\dot{\alpha}_{i-1} & =\sum_{j=1}^{i} \frac{\partial \alpha_{i-1}}{\partial x_{j}} \dot{x}_{j}=\sum_{j=1}^{i} \frac{\partial \alpha_{i-1}}{\partial x_{j}} \theta_{j}^{T} \psi_{j}\left(\bar{x}_{j}, x_{j+1}\right) \\
\dot{z}_{i} & =f_{i}\left(\bar{x}_{i}, x_{i+1}\right)-\dot{\alpha}_{i-1}-b_{i} x_{i+1}+b_{i} x_{i+1} \\
& =\left[\theta_{i, a}^{T} \psi_{i, a}\left(\bar{x}_{i}, x_{i+1}\right)-b_{i} x_{i+1}+b_{i} \alpha_{i}\right]+b_{i} z_{i+1}
\end{aligned}
$$

Note that the intermediate implicit control given in (7) has the property

$$
\frac{\partial \alpha_{i}}{\partial x_{i+1}}=-\frac{1}{b_{i}}\left(1-\frac{\partial \alpha_{i-1}}{\partial x_{i}}\right) \theta_{i}^{T} \frac{\partial \psi_{i}\left(\bar{x}_{i}, x_{i+1}\right)}{\partial x_{i+1}}+1
$$

Accordingly, the Lyapunov function candidate given in (6), has time derivative along (7) and (10) as $\dot{V}_{i}=-\sum_{j=1}^{i} k_{j} z_{j}^{2}+b_{i} z_{i} z_{i+1}$, in which the 
coupling term $b_{i} z_{i} z_{i+1}$ will be handled in the next steps.

Step $n$. In the last step, as $z_{n}=x_{n}-\alpha_{n-1}$ and $\alpha_{n-1}$ is a function of $x$, we have

$$
\begin{aligned}
\dot{\alpha}_{n-1} & =\sum_{i=1}^{n} \frac{\partial \alpha_{n-1}}{\partial x_{j}} \dot{x}_{j}=\sum_{i=1}^{n} \frac{\partial \alpha_{n-1}}{\partial x_{j}} \theta_{j}^{T} \psi_{j}\left(\bar{x}_{j}, x_{j+1}\right) \\
\dot{z}_{n} & =\left[\theta_{n, a}^{T} \psi_{n, a}(x, u)-b_{n} u\right]+b_{n} u
\end{aligned}
$$

Consider the Lyapunov function given in (6). Its time derivative along (8) and (10) is $\dot{V}_{n}=$ $-\sum_{i=1}^{n} k_{i} z_{i}^{2} \leq 0$. Therefore, we can conclude that the closed-loop system is stable provided that there exists bounded solution $u$ for the following equation

$$
-b_{n-1} z_{n-1}-k_{n} z_{n}-\theta_{n, a}^{T} \psi_{n, a}(x, u)=0
$$

The existence of the solution for $u_{1}$ of equation (11) could be guaranteed provided that

$$
\frac{\partial}{\partial u}\left[\theta_{n, a}^{T} \psi_{n, a}(x, u)\right] \neq 0
$$

From the definition of $\psi_{n, a}$, we have

$$
\frac{\partial}{\partial u}\left[\theta_{n, a}^{T} \psi_{n, a}(x, u)\right]=\theta_{n, a}^{T}\left(1-\frac{\partial \alpha_{n-1}}{\partial x_{n}}\right) \frac{\partial \psi_{n}(x, u)}{\partial u}
$$

As $\frac{\partial f_{n}(x, u)}{\partial u} \neq 0$, which is the controllability condition, and $\frac{\partial f_{n}(x, u)}{\partial u}=\theta_{n}^{T} \frac{\partial \psi_{n}(x, u)}{\partial u}$, we know that $(12)$ holds provided that $\left(1-\frac{\partial \alpha_{n-1}}{\partial x_{n}}\right) \neq 0$. From (7), we know that this is equivalent to

$$
\theta_{n-1}^{T}\left(1-\frac{\partial \alpha_{n-2}}{\partial x_{n-1}}\right) \frac{\partial \psi_{n-1}\left(\bar{x}_{n-1}, x_{n}\right)}{\partial x_{n}} \neq 0 .
$$

As $\frac{\partial f_{n-1}\left(\bar{x}_{n-1}, x_{n}\right)}{\partial x_{n}} \neq 0$, which is the controllability condition, and $\frac{\partial f_{n-1}}{\partial x_{n}}=\theta_{n-1}^{T} \frac{\partial \psi_{n-1}}{\partial x_{n}}$, we know that (14) holds provided that $\left(1-\frac{\partial \alpha_{n-2}}{\partial x_{n-1}}\right) \neq$ 0 . Following the similar analysis backwards by noting that $\frac{\partial f_{i}}{\partial x_{i+1}}=\theta_{i}^{T} \frac{\partial \psi_{i}}{\partial x_{i+1}} \neq 0$, we obtain that (12) holds provided that $\left(1-\frac{\partial \alpha_{1}}{\partial x_{2}}\right) \neq 0$ which is actually equivalent to $\theta_{1}^{T} \frac{\partial \psi_{1}}{\partial x_{2}} \neq 0$, which is surely satisfied as it is the controllability condition of the original system.

Theorem 2. For system (1) satisfying Assumptions 1 and 2, implicit control (8) guarantees that $x_{1} \rightarrow 0$ as $t \rightarrow \infty$, the control signal $u$ exists and bounded, and all other signals in the closed-loop are bounded.

In the ideal case that $\theta_{i}$ are known, the advantage of the implicit control lies in that the control $u$ can be easily solved by (8) in digital implementation while it is usually difficult to obtain the explicit solution from (11). In addition, the existence of the solution for $u$ is again guaranteed by the controllability condition for the higherorder nonaffine systems, and there is no additional condition regarding to the existence compared to its first-order counterpart. When the parameters are unknown, the control problem becomes much more complex, and only very restrictive classes of systems can be handled. Due to space limitation, it is omitted here.

\section{APPLICATION TO AFFINE NONLINEAR SAMPLED DATA SYSTEMS}

The implicit controller derived in this paper is a function of not only the states, but also the control input itself, and thus has a form naturally suited for digital implementation in sampled data systems, given mild conditions of smoothness of input. In this section, we illustrate this concept clearly with a simple first order nonlinear system, given by

$$
\dot{x}=a x^{2}+g u=f(x, u)+b u
$$

where $f(x, u):=a x^{2}+g u-b u=\theta^{T} \psi(x, u)-b u$ with $\theta=[a, g]^{T}$, and $\psi(x, u)=\left[x^{2}, u\right]^{T}$; constant $g>0$ to satisfy controllability of the system; and constant $b>0$ is a design control parameter whose role in the stability of the sampled data system will be investigated in this section. We will consider two cases - where the parameter $\theta$ is known, and where it is unknown.

\subsection{Known Case}

For simplicity, let us consider the case that $f(x, u)$ is known first. From (15), we know that

$$
u=\frac{1}{b}(-f(x, u)-c x), \quad b, c>0
$$

is stabilizing as it renders the closed-loop system as $\dot{x}=-c x$.

In the analogue world, we could indeed assume that $u(k+1)=u(k)$, where $u(k+1)$ and $u(k)$ denote $u\left(\tau_{k+1}\right)$ and $u\left(\tau_{k}\right)$, respectively. Accordingly, stable control (16) could be easily implemented as

$$
u(k+1)=\frac{1}{b}\left(-a x^{2}(k)-g u(k)+b u(k)-c x(k)\right)
$$

where $x(k)$ denotes $x\left(\tau_{k}\right) ; u(k+1)$ and $u(k)$ denote the control signals of the next and current sampling intervals, respectively.

For digital implementation, implementation (17) is not necessarily equivalent to feedback linearization because $u(k+1) \neq u(k)$ in general. However, 
under the assumption that $u(t)$ is smooth enough, the error might be acceptable and the closed-loop system is stable under certain mild conditions.

As the resulting closed system is indeed a hybrid system, its stability should be analyzed in detail using the stability criterion for sampled data systems. In fact, the closed-loop hybrid system is given by

$$
\begin{aligned}
\dot{x}(t) & =a x^{2}(t)+g u\left(\tau_{k}\right), \tau_{k} \leq t<\tau_{k+1} \\
u\left(\tau_{k+1}\right) & =\left(1-\frac{g}{b}\right) u\left(\tau_{k}\right)-\frac{a}{b} x^{2}\left(\tau_{k}\right)-\frac{c}{b} x\left(\tau_{k}\right)
\end{aligned}
$$

Its local stability can be investigated for the linearized system. Define $f(x, u)=a x^{2}+g u$ and $g(x, u)=\left(1-\frac{g}{b}\right) u-\frac{a}{b} x^{2}-\frac{c}{b} x$.

Linearizing the system (19) about the point $\left(x_{e}, u_{e}\right)=(0,0)$, we obtain the system

$$
\begin{aligned}
& \dot{y}(t)=\left[\begin{array}{ll}
A & B \\
0 & 0
\end{array}\right] y(t)+\tilde{F}(y(t)), \tau_{k} \leq t<\tau_{k+1}, \quad(19) \\
& y(t)=\left[\begin{array}{ll}
I & 0 \\
D & C
\end{array}\right] y\left(t^{-}\right)+\tilde{H}\left(y\left(t^{-}\right)\right), t=\tau_{k+1}, k \in N
\end{aligned}
$$

where $A=0, B=g, C=1-\frac{g}{b}, D=-\frac{c}{b}$, and

$$
\lim _{y \rightarrow 0} \frac{\|\tilde{F}(y)\|}{\|y\|}=0, \quad \lim _{y \rightarrow 0} \frac{\|\tilde{H}(y)\|}{\|y\|}=0
$$

According to Theorem 2.2 in (Michel and $\mathrm{Hu}$, 1999), if $\left\|U_{k}\right\| \leq 1$, the system (19) is uniformly asymptotically stable. We have $\left\|U_{k}\right\|=\left|1-\frac{g}{b}\right|<1$ which leads to the stability condition $0<\frac{g}{b}<2$.

Note that the larger the $b$ chosen, the more stable the system will be. This condition will be verified by the following simulation studies.

Simulation Studies Consider the same system as (15) with $a=3$ and $g=2$. The feedback gain is chosen as $c=2.0$. The initial condition is $x(0)=$ 2. According to the condition for bounded control input, $0<g / b<2$, i.e., $b>1$. For comparison, simulations are conducted for $b=1.01, b=1$, and $b=0.99$. Fig. 1 shows the trajectory of the state and Fig. 2 plots the control input. From Fig. 1 and Fig. 2, it can be seen that the choice of $b$ is critical. When $b$ is chosen as $b \leq 1$, the system state does not converge to the origin and the control input takes large values.

\subsection{Unknown Case}

For comparsion, let us consider the case that the parameters are unknown constants. Define sampling interval $T=\tau_{k+1}-\tau_{k}$. A feasible implementation of the parameter estimation law is

$$
\begin{aligned}
& \hat{a}\left(\tau_{k+1}\right)=\hat{a}\left(\tau_{k}\right)+T \gamma_{1} x^{3}\left(\tau_{k}\right) \\
& \hat{g}\left(\tau_{k+1}\right)=\hat{g}\left(\tau_{k}\right)+T \gamma_{2} x\left(\tau_{k}\right) u\left(\tau_{k}\right)
\end{aligned}
$$

where $\gamma_{1}, \gamma_{2}>0$. Noting that $\tilde{a}=a-\hat{a}, \tilde{g}=g-\hat{g}$, the closed-loop system can be written as

$$
\begin{aligned}
\dot{x}(t)= & a x^{2}(t)+g u\left(\tau_{k}\right) \\
u\left(\tau_{k+1}\right)= & \left(1-\frac{g-\tilde{g}\left(\tau_{k}\right)}{b}\right) u\left(\tau_{k}\right)-\frac{a-\tilde{a}\left(\tau_{k}\right)}{b} x^{2}\left(\tau_{k}\right) \\
& -\frac{c}{b} x\left(\tau_{k}\right)
\end{aligned}
$$

Following the same procedure, linearizing the system about the equilibrium point $\left(x_{e}, \tilde{a}_{e}, \tilde{g}_{e}, u_{e}\right)=$ $(0,0,0,0)$, we can obtain $\left\|U_{k}\right\|=\left|\left(1-e^{T}\right)^{2}\left(1-\frac{g}{b}\right)\right|$ i.e.,

$$
b<\frac{g}{1-\frac{1}{\left(1-e^{T}\right)^{2}}} \quad \text { or } \quad b>\frac{g}{1+\frac{1}{\left(1-e^{T}\right)^{2}}}
$$

It is clear that the stability of the adaptive controller is directly influenced by the size of the sampling interval, $T$, in the local sense. This also verifies the fact that, as the complexity of the system increases, more stringent requirements are expected and much more complex behavior will be observed.

However, it is found in the simulation that the closed-loop system becomes unstable for certain choices of $b$ satisfying the condition in (22). Therefore, for such $b$ satisfying (22), although the closed-loop stability is guaranteed for the linearized system characterized by $(A, B, C, D)$, this is not the case for its original hybrid system (21). Further analysis should be done!

Simulation Studies Consider the same system as (15). From the system parameters, let us choose $b=3.1, b=0.8005$ and $b=10$ respectively. The trajectories of state and control input are illustrated in Fig. 3 and Fig. 4. It is found that the system is unstable for $b<0.8$. The convergence of the parameter estimations for $b=3.1$ is shown in Fig. 5.

\section{CONCLUSION}

Implicit control design has been presented for different classes of nonlinear systems, including nonaffine systems in pure-feedback form and affine systems in strict-feedback form. The differences between the proposed method and the feedback linearization based control have been stated. The stability issue arising from the digital implementation of the proposed control, which has resulted in a hybrid system, has been discussed and the stability condition has been given. Simulation studies show the validity of the proposed design. 


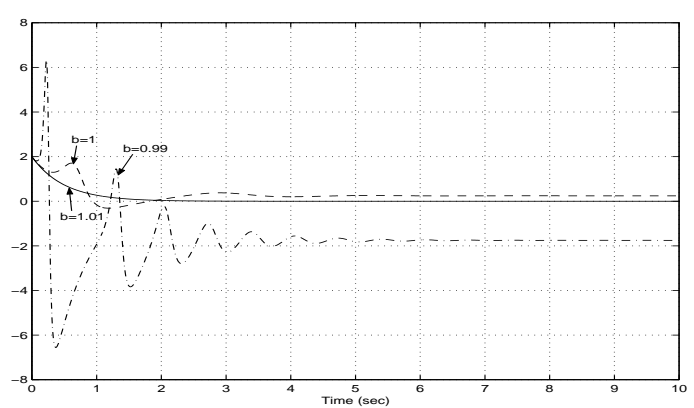

Fig. 1. Trajectory of state $x(t): b=1.01$ ("-"), $b=1$ ("-"), $b=0.99$ (" .").

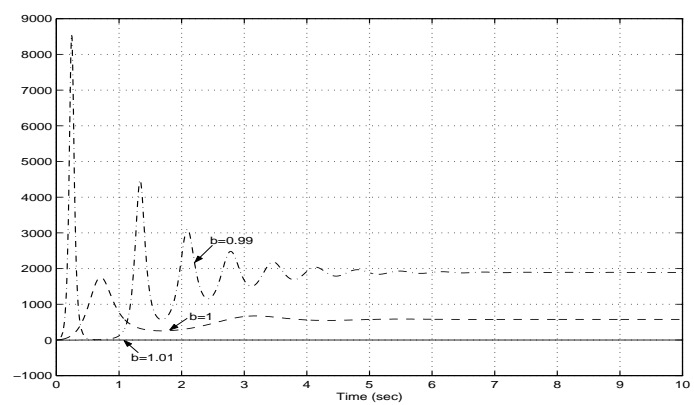

Fig. 2. Control input $u(t): b=1.01$ ("-"), $b=1$ ("- -"), $b=0.99$ ("-.").

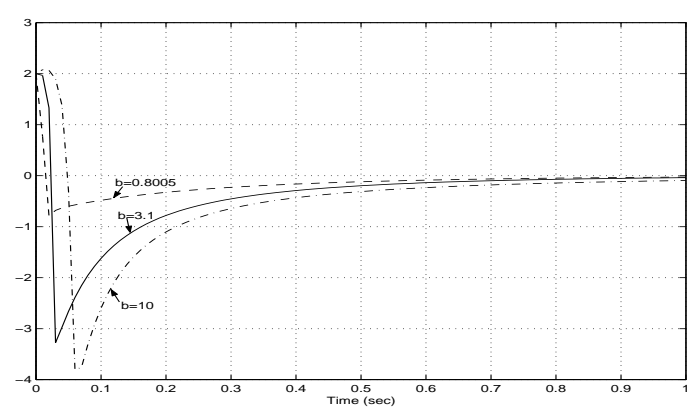

Fig. 3. Trajectory of state $x(t): b=3.1($ " " ),$\quad b=$ 0.8005 ("- -"), $b=10$ ("“- .").

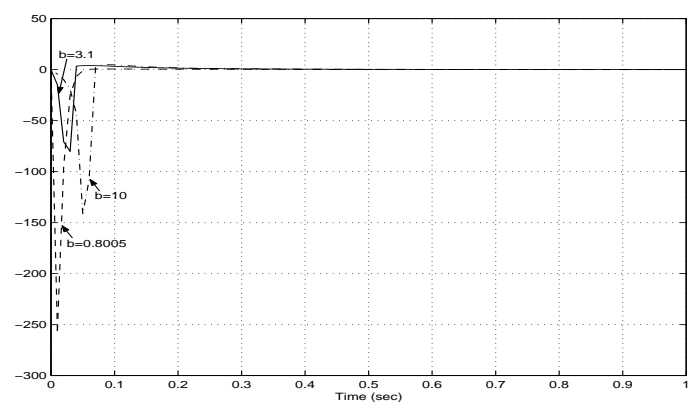

Fig. 4. Control input $u(t): b=3.1$ ("-"), $b=0.8005$ ("-"), $b=10$ ("- .").

\section{REFERENCES}

Ge, S. S., C. C. Hang and T. Zhang (1999a). Adaptive neural network control of nonlinear systems by state and output feedback. IEEE Trans. Syst., Man, Cybern. B 29(6), 818-828.

Ge, S. S., C. C. Hang and T. Zhang (1999b). Nonlinear adaptive control using neural networks and its application to cstr systems. Journal of Process Control 9(4), 313-323.

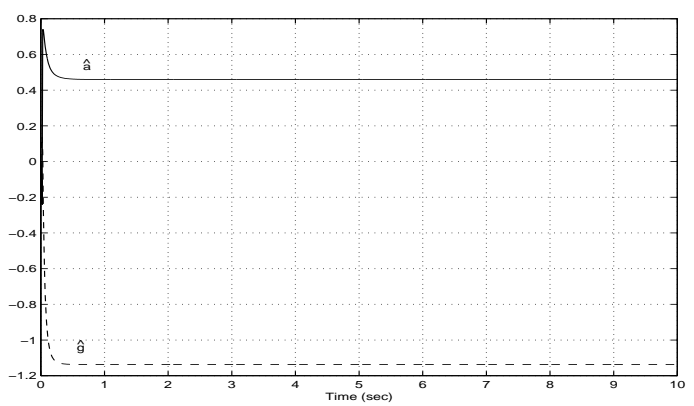

Fig. 5. Parameter estimation $\hat{a}$ ("-") and $\hat{g}$ (“- -").

Goh, C. J. (1994). Model reference control of nonlinear systems via implicit function emulation. Int. J. Control 60, 91-115.

Goh, C. J. and T. H. Lee (1994). Direct adaptive control of nonlinear systems via implicit function emulation. Control-Theory and Advance Technology 10(3), 539-552.

Hovakimyan, N., F. Nardi, A. Calise and K. Nakwan (2002). Adaptive output feedback control of uncertain nonlinear systems using single-hidden-layer neural networks. IEEE Trans. Neural Networks 13(6), 1420-1431.

Jin, L., P. N. Nikiforuk and M. M. Gupta (1993). Direct adaptive output tracking control using multilayered neural networks. IEE Proc. D 140(6), 393-398.

Kanellakipoulos, I., P. V. Kokotović and A. S. Morse (1991). Systematic design of adaptive controllers for feedback linearizable systems. IEEE Trans. Automat. Contr. 36(11), 12411253.

Krstić, M., I. Kanellakopoulos and P. V. Kokotović (1995). Nonlinear and Adaptive Control Design. Wiley. New York.

Levin, A. U. and K. S. Narendra (1996). Control of nonlinear dynamical systems using neural networks - part ii: observability, identification, and control. IEEE Trans. Neural Networks 7(1), 30-42.

Michel, A. N. and B. Hu (1999). Towards a stability theory of general hybrid dynamical systems. Automatica 35(3), 371-384.

Nesic, D., E. Skafidas, I.M.Y. Mareels and R.J. Evans (1999). Minimum phase properties for input nonaffine nonlinear systems. IEEE Trans. Automat. Contr. 44(4), 868-872.

Seto, D., A. M. Annaswamy and J. Baillieul (1994). Adaptive control of nonlinear systems with a triangular structure. IEEE Trans. Automat. Contr. 39(7), 1411-1428.

Simoes, C., H. Nijmeijer and J. Tsinias (1996). Nonsmooth stabilizability and feedback linearization of discrete-time nonlinear systems. Int. J. Robust and Nonlinear Contr. 6(3), 171-188. 